Research Paper

\title{
Frequency and Clinical Characteristics of Unselected Korean Gastric Cancer Patients with a Germline CDHI V832M Mutation
}

\author{
Saeam Shin ${ }^{1^{*}}$, Yoonjung $\mathrm{Kim}^{1^{*}}$, Jin Kyung Lee ${ }^{2,3 凶}$, Kyung-A Lee ${ }^{1^{凶}}$ \\ 1. Department of Laboratory Medicine, Yonsei University College of Medicine, Seoul, Korea; \\ 2. Department of Laboratory Medicine, Korea Cancer Center Hospital, Korea Institute of Radiological and Medical Sciences, Seoul, Korea; \\ 3. KIRAMS Radiation Biobank, Korea Institute of Radiological and Medical Sciences, Seoul, Korea. \\ *These authors contributed equally to this work.
}

$\triangle$ Corresponding authors: Kyung-A Lee, MD, PhD, Department of Laboratory Medicine, Yonsei University College of Medicine, 211, Eonju-ro, Gangnam-gu, Seoul 06273, Korea. KAL1119@yuhs.ac; Telephone: +82-2-2019-3531; Fax: +82-2-2019-4822 or Jin Kyung Lee, MD, PhD, KIRAMS Radiation Biobank, Korea Institute of Radiological and Medical Sciences, 75 Nowon-ro, Nowon-gu, Seoul 01812, Korea. jklee@kirams.re.kr; Telephone: +82-2-970-2492; Fax: +82-2-973-7143

(C) The author(s). This is an open access article distributed under the terms of the Creative Commons Attribution License (https://creativecommons.org/licenses/by/4.0/). See http://ivyspring.com/terms for full terms and conditions.

Received: 2019.05.08; Accepted: 2019.09.21; Published: 2020.01.01

\begin{abstract}
Background: Germline mutations in $\mathrm{CDHI}$ are associated with hereditary and early onset- diffuse gastric cancer. However, the frequency of $\mathrm{CDHI}$ germline mutation in unselected gastric cancer cases is not well established.

Aim: The aim of this study was to investigate the frequency and clinical characteristics of germline $\mathrm{CDHI}$ V832M mutation carriers in unselected Korean gastric cancer cases.

Methods: Direct sequencing was performed to determine the presence of $C D H I$ V832M in 305 unselected Korean gastric cancer patients. Lauren's histologic type, family history of gastric cancer, and age of cancer diagnosis were compared between V832M carriers and non-carriers.

Results: In the study population, seven gastric cancer patients $(7 / 305,2.29 \%)$ were found to have the $C D H I$ V832M mutation. The CDHI V832M mutation carrier state was not significantly associated with phenotypes including Lauren's histologic type, family history of gastric cancer, age of cancer diagnosis, and other cancer history in a patient.

Conclusion: This study demonstrates that the germline $\mathrm{CDHI}$ V832M mutation is common in sporadic, late onset, and intestinal type gastric cancer as well as familial, early onset, and diffuse type gastric cancer. Our finding suggests that guidelines for managing $\mathrm{CDHI}$ mutation carriers should be refined through additional data on penetration according to $\mathrm{CDHI}$ mutation type in sporadic cases.
\end{abstract}

Key words: $C D H 1$, E-cadherin, gastric cancer, hereditary gastric cancer, germline mutation

\section{Introduction}

Hereditary diffuse gastric cancer (HDGC, OMIM $\# 137215)$ is an autosomal dominant cancer predisposition syndrome. Germline mutation in the E-cadherin gene $(C D H 1)$ is associated with familial cases of diffuse type gastric cancer and lobular breast cancer [1]. Patients with HDGC characteristically develop diffuse gastric cancer at a young age; the average age for gastric cancer diagnosis is 37 years [2]. The cumulative risk of gastric cancer for pathogenic $\mathrm{CDH1}$ mutation carriers by age 80 years is estimated to be $70 \%$ ( $95 \%$ CI, $59 \%$ to $80 \%$ ) for males and $56 \%$ (95\% CI, $44 \%$ to $69 \%$ ) for females [1]. Because of the high mortality from invasive cancer, early prophylactic total gastrectomy is recommended for CDH1 truncating mutation carriers in current clinical guidelines [2,3]. However, information about penetration and cancer risk of missense mutations, which accounts for $30 \%$ of total mutations found in HDGC families, is still insufficient [4]. Moreover, as panel testing using next-generation sequencing 
increases in popularity, the likelihood of detecting CDH1 germline mutations in patients not meeting HDGC diagnostic criteria is increasing and genetic counseling for these patients with missense mutations would confer a significant clinical burden $[5,6]$.

Recently, we identified a CDH1 V832M [NM_004360.4:c.2494G>A， NP_004351.1:p.(V832M)] missense variant in two out of 107 unselected Korean patients with gastric cancer $(2 / 107,1.87 \%)$ [7]. They were diagnosed with late-onset gastric cancer at 66 and 75 years, respectively. The V832M variant has been identified in a Japanese HDGC family, and the three-affected members of the family had the same mutation [8]. The mutation has been shown to disrupt the ability of E-cadherin to mediate cell adhesion and to suppress cell invasion in previous functional studies [9-11].

Based on these findings, we hypothesized that the CDH1 V832M mutation would be prevalent in Korean sporadic gastric cancer patients. The objective of this study was to assess the frequency of CDH1 V832M mutation carriers in unselected Korean gastric cancer patients with a high incidence of sporadic gastric cancer. We investigated whether gastric cancer patients with a $C D H 1$ pathogenic missense mutation, V832M, actually showed characteristics of HDGC; familial gastric cancer, early-onset gastric cancer, and diffuse histology.

\section{Materials and Methods}

\section{Patient and samples}

Samples from 305 Korean patients who were histologically diagnosed with gastric cancer were provided by the Korea Institute of Radiological and Medical Sciences (KIRAMS) Radiation Biobank (KRB), Yonsei University Wonju Medical Center Biobank, and Samkwang Medical Laboratory Biobank. This study protocol was approved by the institutional review board. The samples were unselected for age at diagnosis and family history of gastric cancer. Clinical information including patient demographics, tumor histopathology, and family history of cancer was provided from the sample source institutions. The tumor histology was classified according to Lauren's criteria [12] and the 2010 WHO classification system [13]. The stage of tumor was assigned according to the 7th edition of the American Joint Committee on Cancer TNM classification system [14]. Information about family history of cancer and other cancer histories in a patient was obtained from 198 patients. A family history of gastric cancer is defined as a gastric cancer patient having one or more first- or second-degree relatives with gastric cancer. Earlyonset gastric cancer is defined as a gastric cancer patient diagnosed at or before the age of 45 years; late-onset gastric cancer was defined as gastric cancer patients diagnosed over the age of 45 years.

\section{CDH1 V832M genotyping}

Genomic DNA was extracted from buffy-coats or normal tissues using the QIAamp DNA Mini kit (Qiagen, Hilden, Germany). The primer sequences targeting codon 814 to 919 of CDH1 exon 16 were designed using Primer3 software. The PCR was performed on $100 \mathrm{ng}$ of genomic DNA, and sequencing was done with the Big Dye Terminator v3.1 Cycle Sequencing Ready Reaction Kit (Applied Biosystems, Foster City, CA, USA) on the ABI 3500 Genetic Analyzer (Applied Biosystems). The results were compared with the reference sequence using Sequencher 5.1 software (Gene Codes Corp., Ann Arbor, MI, USA).

\section{Statistical analysis}

Statistical analysis was performed using SPSS Statistics version 24.0.0 (IBM Corp., Armonk, NY, USA). For comparison between mutation carriers and non-carriers, we used Fisher's exact test for categorycal variables and the Mann-Whitney $U$ test for continuous variables. All $p$-values were two-tailed, and values less than 0.05 were considered statistically significant.

\section{Results}

Clinicopathological data for 305 unselected Korean gastric cancer patients are summarized in Table 1. The study included 194 males and 111 females, with a median age of 64 years (range, 31-91 years). This series included 227 intestinal types (74.4\%), 56 diffuse types (18.4\%), and 22 mixed types $(7.2 \%)$ in accordance with Lauren's classification. According to the AJCC/UICC criteria, there were 151 cases in stage I (49.5\%), 55 cases in stage II (18.0\%), 83 cases in stage III $(27.2 \%)$, and 16 cases $(5.2 \%)$ in stage IV. Among 198 patients with information about a family history and history of cancer, 37 patients $(18.7 \%)$ had family history of cancer in first- or second- degree relatives and 13 patients had a past history of cancer other than gastric cancer $(6.6 \%)$. Early-onset cases (age of cancer diagnosis $\leq 45$ years) represented $9.5 \%$ of total participants.

In the present study, we found seven carriers of the $C D H 1$ V832M mutation (carrier frequency = $0.0230,7 / 305$ ) (Table 2). The carrier frequencies peaked at $60-69$ years $(0.0361)$, followed by $50-59$ years (0.0274) (Table 3). There was one case (P186) of early-onset ( $\leq 45$ years) cancer with the V832M mutation. The patient was diagnosed with intestinaltype gastric adenocarcinoma at 41 years and had a 
family history of gastric cancer in his father. There were two cases (P41 and P253) with diffuse-type cancer histology and V832M mutation, and neither case had a known family history of gastric cancer.

We compared the known phenotypic characteristics of HDGC by V832M carrier status (Table 4). The median age of cancer diagnosis was not different among V832M carriers vs. non-carriers (62 vs. 64 years, respectively, $P$ value $=0.477$, Mann-Whitney $U$ test). The diffuse or mixed type histology based on Lauren's classification was not significantly associated with the V832M carrier status ( $p$-value $>0.999$, Fisher's exact test). Moreover, a family history of gastric cancer, age of cancer diagnosis, and other cancer histories in a patient was also not significantly associated with the V832M carrier status.

\section{Discussion}

The $C D H 1$ gene encodes an epithelial cadherin protein, E-cadherin. E-cadherin plays roles in regulating cell-cell adhesion, mobility and proliferation of epithelial cells [15]. Loss of function of E-cadherin impairs epithelial cell adhesiveness and contributes to cancer progression and metastasis [16]. The germline pathogenic truncating and missense mutations are distributed throughout the entire $\mathrm{CDH} 1$ gene, and the V832M mutation is located in exon 16, the cytoplasmic domain of the protein [17]. The V832M mutation was first identified in a Japanese HDGC family, and was reported again in a patient with early-onset lobular breast cancer $[8,18]$. This missense mutation has been shown to affect the role of E-cadherin in mediating cell adhesion and suppressing cell invasion [9-11].

Current clinical guidelines regarding HDGC recommend prophylactic total gastrectomy for $\mathrm{CDH1}$ pathogenic mutation carriers due to the observation of early gastric cancer in prophylactic gastrectomy samples from $C D H 1$ mutation carriers [2,3]. $C D H 1$ germline mutations have been described in about $40 \%$ of HDGC families and about $2.3 \%$ of sporadic early-onset gastric cancer patients [1]. Recently, Lo et al [17] reported that the location and type of germline CDH1 variants are related to the age of cancer onset and risk of concomitant cancer in the HDGC families. However, data on the frequency and clinical characteristics of germline CDH1 mutations in unselected gastric cancer patients are scarce. Due to limited data, policies for genetic counseling and management in germline $\mathrm{CDH} 1$ mutation carriers in sporadic gastric cancer have not been established. As panel testing increases in popularity, $\mathrm{CDH1}$ variants might be identified in patients who do not meet HDGC criteria $[6,7,19]$. Huynh et al. reported two families with $C D H 1$ mutations that did not meet any of the International Gastric Cancer Linkage Consort- ium (IGCLC) criteria [6]. The two families with a nonsense mutation and a splicing mutation in $C D H 1$, respectively, did not have a family history of diffuse gastric cancer [6]. These findings suggest that a different approach is needed when a CDH1 mutation is found in a sporadic or asymptomatic setting.

Frequencies of $\mathrm{CDH1}$ germline mutations in gastric cancer have been known to vary according to the incidence of gastric cancer $[3,20]$. Corso et al. reported that areas with a high risk of gastric cancer show a higher frequency of $C D H 1$ missense mutations than non-missense mutations [20]. Current clinical guidelines are based primarily on data from areas with a low-incidence of sporadic gastric cancer and truncating mutations [3]. Based on our prior observation [7], we searched the V832M mutation in unselected Korean gastric cancer patients, and the mutation was found regardless of the age of cancer diagnosis, family history of gastric cancer, or Lauren's histology type.

Table 1. Clinicopathological characteristics of patients with gastric cancer

\begin{tabular}{|c|c|}
\hline Variables & All patients $(N=305)^{a}$ \\
\hline Age (years) & $64(53.5,74)$ \\
\hline \multicolumn{2}{|l|}{ Sex } \\
\hline Female & $111(36.4 \%)$ \\
\hline Male & $194(63.6 \%)$ \\
\hline \multicolumn{2}{|l|}{ Lauren's classification } \\
\hline Diffuse & $56(18.4 \%)$ \\
\hline Intestinal & $227(74.4 \%)$ \\
\hline Mixed & $22(7.2 \%)$ \\
\hline \multicolumn{2}{|l|}{ WHO classification } \\
\hline Mucinous & $5(1.6 \%)$ \\
\hline Tubular & $239(78.4 \%)$ \\
\hline Poorly cohesive & $57(18.7 \%)$ \\
\hline Mixed (tubular and poorly cohesive) & $2(0.7 \%)$ \\
\hline Mixed (tubular and papillary) & $1(0.3 \%)$ \\
\hline Adenosquamous & $1(0.3 \%)$ \\
\hline \multicolumn{2}{|l|}{ TNM classification } \\
\hline Stages IA/IB & $114(37.4 \%) / 37(12.1 \%)$ \\
\hline Stages IIA/IIB & $30(9.8 \%) / 25(8.2 \%)$ \\
\hline Stages IIIA/IIIB/IIIC & $34(11.1 \%) / 22(7.2 \%) / 27(8.9 \%)$ \\
\hline Stages IV & $16(5.2 \%)$ \\
\hline \multicolumn{2}{|l|}{ Family history of gastric cancer } \\
\hline 2 family members & $5(1.6 \%)$ \\
\hline 1 family member & $32(10.5 \%)$ \\
\hline No family history & $161(52.8 \%)$ \\
\hline Unknown & $107(35.1 \%)$ \\
\hline \multicolumn{2}{|l|}{ Age of cancer diagnosis } \\
\hline Early-onset ( $\leq 45$ years) & $29(9.5 \%)$ \\
\hline Late-onset (>45 years) & $276(90.5 \%)$ \\
\hline \multicolumn{2}{|l|}{ Other cancer histories in a patient } \\
\hline Breast cancer & $2(0.7 \%)$ \\
\hline Colon cancer & $3(1.0 \%)$ \\
\hline Other cancerb & $8(2.6 \%)$ \\
\hline None & $185(60.7 \%)$ \\
\hline Unknown & $107(35.1 \%)$ \\
\hline
\end{tabular}

a Results are expressed as median (interquartile ranges) or number (\%). ${ }^{\mathrm{b} T w o}$ patients with urinary tract cancer, a patient with essential thrombocythemia, a patient with pharyngeal cancer, a patient with lung cancer, a patient with papillary thyroid cancer, a patient with ovary cancer, and a patient with hepatocellular carcinoma and cervix cancer. 
Table 2. Korean gastric cancer patients with CDHI V832M germline mutation identified in this study

\begin{tabular}{|c|c|c|c|c|c|c|c|c|c|c|}
\hline ID & Sex & $\begin{array}{l}\text { Age at } \\
\text { diagnosis }\end{array}$ & $\begin{array}{l}\text { Lauren's } \\
\text { classification }\end{array}$ & $\begin{array}{l}\text { WHO } \\
\text { classification }\end{array}$ & $\begin{array}{l}\text { TNM } \\
\text { classification }\end{array}$ & $\begin{array}{l}\text { EBV } \\
\text { infection }{ }^{a}\end{array}$ & $\begin{array}{l}\text { MSI } \\
\text { state }^{b}\end{array}$ & $\begin{array}{l}\text { H. pylori } \\
\text { infectionc }\end{array}$ & $\begin{array}{l}\text { Family history of } \\
\text { cancer (affected family } \\
\text { member) }\end{array}$ & $\begin{array}{l}\text { Other cancer histories in a } \\
\text { patient (age at diagnosis) }\end{array}$ \\
\hline P41 & $\mathrm{F}$ & 50 & Diffuse & Poorly cohesive & Stage IA & Negative & MSS & Negative & None & Papillary thyroid cancer (49) \\
\hline P93 & $\mathrm{F}$ & 58 & Intestinal & Tubular & Stage IA & Negative & MSS & Not done & Colon cancer (a brother) & None \\
\hline P119 & M & 62 & Intestinal & Tubular & Stage IIIA & Positive & MSS & Not done & None & None \\
\hline P143 & $\mathrm{F}$ & 66 & Intestinal & Tubular & Stage IIA & Negative & MSI-H & Not done & Lymphoma (a son) & None \\
\hline P154 & M & 66 & Intestinal & Tubular & Stage IIB & Negative & MSS & Positive & Unknown & Unknown \\
\hline P186 & M & 41 & Intestinal & Tubular & Stage IA & Negative & MSS & Negative & Gastric cancer (a father) & None \\
\hline P253 & M & 75 & Diffuse & Tubular & Stage IIB & Negative & MSI-H & Negative & Unknown & Unknown \\
\hline
\end{tabular}

EBV, Epstein-Barr virus; MSI, microsatellite instability; H. pylori, Helicobacter pylori; MSS, microsatellite stability; MSI-H, microsatellite instability high. aEBV infection was

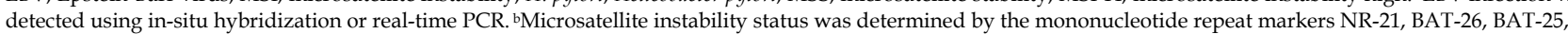
NR-24, and NR-27. MSI-H was defined as a tumor with two or more of the five markers of instability, and MSS was defined as the absence of any marker. ${ }^{2}$. Pylori infection was detected using Giemsa stain or PCR and sequencing.

Table 3. Frequencies of $\mathrm{CDH} 1 \mathrm{~V} 832 \mathrm{M}$ mutation as related to the age at gastric cancer diagnosis

\begin{tabular}{lll}
\hline Age at diagnosis & No. of patients & No. of carriers (carrier frequency) \\
\hline$<40$ & 6 & 0 \\
$40-49$ & 43 & $1(0.0233)$ \\
$50-59$ & 73 & $2(0.0274)$ \\
$60-69$ & 83 & $3(0.0361)$ \\
$70-79$ & 78 & $1(0.0128)$ \\
$\geq 80$ & 22 & 0 \\
Total & 305 & $7(0.0230)$ \\
\hline
\end{tabular}

Table 4. Association of the CDHI V832M germline mutation and phenotypes in gastric cancer patients

\begin{tabular}{|c|c|c|c|}
\hline Variables & $\begin{array}{l}\text { V832M } \\
\text { heterozygotes } \\
(N=7)^{a}\end{array}$ & $\begin{array}{l}\text { V832M } \\
\text { non-carrier } \\
(N=298)^{a}\end{array}$ & $\begin{array}{l}P \\
\text { value }^{b}\end{array}$ \\
\hline Age (years) & $62(54,66)$ & $64(54,74)$ & 0.477 \\
\hline Sex & & & 0.708 \\
\hline Female & $3(42.9 \%)$ & $108(36.2 \%)$ & \\
\hline Male & $4(57.1 \%)$ & $190(63.8 \%)$ & \\
\hline Lauren's classification & & & $>0.999$ \\
\hline Diffuse/mixed type & $2(28.6 \%)$ & $76(25.5 \%)$ & \\
\hline Intestinal type & $5(71.4 \%)$ & $222(74.5 \%)$ & \\
\hline Family history of gastric cancerc & & & $>0.999$ \\
\hline $\begin{array}{l}\text { Presence (one or more family } \\
\text { members) }\end{array}$ & $1(20.0 \%)$ & $36(18.7 \%)$ & \\
\hline Absence & $4(80.0 \%)$ & $157(81.3 \%)$ & \\
\hline Age of cancer diagnosis & & & 0.507 \\
\hline Early-onset ( $\leq 45$ years) & $1(14.3 \%)$ & $28(9.4 \%)$ & \\
\hline Late-onset (>45 years) & $6(85.7 \%)$ & $270(90.6 \%)$ & \\
\hline Other cancer histories in a patientc & & & 0.290 \\
\hline Presence & $1(20.0 \%)$ & $12(6.2 \%)$ & \\
\hline Absence & $4(80.0 \%)$ & $181(93.8 \%)$ & \\
\hline
\end{tabular}

The CDH1 V832M allele is common in Koreans and Japanese, with reported population frequencies of $0.016(30 / 1909)$ and $0.013(1 / 76)$, respectively, in the gnomad database (http://gnomad.broadinstitute. org). The expected number of carriers was not different from the numbers observed in this study (5.10 vs. $7, P=0.360, X^{2}$ test). The reason why the V832M mutation is so frequent in the population database is that the variant might have low-penetrance and late-onset characteristics, which encompass many carriers in the control population. To estimate the cancer risk associated with the V832M mutation, further studies using cancer-free age-matched controls are needed.

The limitation of this study is that we could not get information about histology type in cases with a family history of gastric cancer. Since our study was conducted retrospectively and the series had not been fully evaluated for HDGC clinical criteria, HDGC was not completely excluded in our series. Future studies are needed with more patients with a detailed family history.

To date, studies have not evaluated known CDH1 missense mutations in a large number of unselected gastric cancer patients to determine the statistical association with clinical features. We firstly proved that a known missense mutation, V832M, is not associated with clinical features previously known as characteristics of patients with $\mathrm{CDH} 1$ mutation carriers. Especially our study is directly relevant to East-Asians who are common with the V832M allele. It also suggests that the same approach is needed for missense mutations common to other races.

Our study shows that a germline CDH1 V832M mutation is commonly found in sporadic, late onset, or intestinal type gastric cancer, as well as familial, early onset, or diffuse type gastric cancer. These findings suggest that the presence of the V832M mutation is not an indicator of HDGC diagnosis or early-onset of gastric cancer in a patient. Therefore, if the V832M mutation is found in patients who do not meet the HDGC clinical criteria, the risk prediction and management, including preventive surgery, need to be differentiated from that of HDGC patients. It is important to supplement the management protocol based on more data on $\mathrm{CDH1}$ mutation penetrance in sporadic gastric cancer cases among different geographic regions. 


\section{Acknowledgements}

This study was supported by grants of the Korea Institute of Radiological and Medical Sciences (KIRAMS), funded by Ministry of Science and ICT (MSIT), Republic of Korea (No. 50544-2019) and of the National Research Foundation of Korea (NRF-2015R 1C1A2A01055967). The specimens used for this study were distributed by the Korea Institute of Radiological and Medical Sciences (KIRAMS) Radiation Biobank (KRB).

\section{Competing Interests}

The authors have declared that no competing interest exists.

\section{References}

1. Hansford S, Kaurah P, Li-Chang H, et al. Hereditary Diffuse Gastric Cancer Syndrome: CDH1 Mutations and Beyond. JAMA Oncol. 2015;1(1):23-32.

2. Network NCC. NCCN clinical practice guidelines in oncology (NCCN guidelines): gastric cancer. Version 2. 2018. 2018.

3. van der Post RS, Vogelaar IP, Carneiro F, et al. Hereditary diffuse gastric cancer: updated clinical guidelines with an emphasis on germline CDH1 mutation carriers. J Med Genet. 2015;52(6):361-374.

4. Suriano G, Seixas S, Rocha J, et al. A model to infer the pathogenic significance of CDH1 germline missense variants. J Mol Med (Berl). 2006;84(12):1023-1031.

5. Kurian AW, Hare EE, Mills MA, et al. Clinical evaluation of a multiple-gene sequencing panel for hereditary cancer risk assessment. J Clin Oncol. 2014;32(19):2001-2009.

6. Huynh JM, Laukaitis CM. Panel testing reveals nonsense and missense CDH1 mutations in families without hereditary diffuse gastric cancer. Mol Genet Genomic Med. 2016;4(2):232-236.

7. Kim Y, Cho MY, Kim J, et al. Profiling cancer-associated genetic alterations and molecular classification of cancer in Korean gastric cancer patients. Oncotarget. 2017;8(41):69888-69905.

8. Yabuta T, Shinmura K, Tani M, et al. E-cadherin gene variants in gastric cancer families whose probands are diagnosed with diffuse gastric cancer. Int J Cancer. 2002;101(5):434-441.

9. Suriano G, Mulholland D, de Wever O, et al. The intracellular E-cadherin germline mutation V832 M lacks the ability to mediate cell-cell adhesion and to suppress invasion. Oncogene. 2003;22(36):5716-5719.

10. Figueiredo J, Soderberg $\mathrm{O}$, Simoes-Correia $\mathrm{J}$, et al. The importance of E-cadherin binding partners to evaluate the pathogenicity of E-cadherin missense mutations associated to HDGC. Eur J Hum Genet. 2013;21(3):301-309.

11. Bajpai S, Correia J, Feng Y, et al. \{alpha\}-Catenin mediates initial E-cadherin-dependent cell-cell recognition and subsequent bond strengthening. Proc Natl Acad Sci U S A. 2008;105(47):18331-18336.

12. Lauren P. The Two Histological Main Types Of Gastric Carcinoma: Diffuse And So-Called Intestinal-Type Carcinoma. An Attempt At A Histo-Clinical Classification. Acta Pathol Microbiol Scand. 1965;64:31-49.

13. Bosman FT, International Agency for Research on C. WHO classification of tumours of the digestive system. Lyon: IARC; 2010.

14. Washington K. 7th edition of the AJCC cancer staging manual: stomach. Ann Surg Oncol. 2010;17(12):3077-3079.

15. Takeichi M. Cadherin cell adhesion receptors as a morphogenetic regulator. Science. 1991;251(5000):1451-1455.

16. Christofori G, Semb H. The role of the cell-adhesion molecule E-cadherin as a tumour-suppressor gene. Trends Biochem Sci. 1999;24(2):73-76.

17. Lo $\mathrm{W}$, Zhu B, Sabesan A, et al. Associations of CDH1 germline variant location and cancer phenotype in families with hereditary diffuse gastric cancer (HDGC). J Med Genet. 2019; doi:10.1136/jmedgenet-2018-105361.

18. Schrader KA, Masciari S, Boyd N, et al. Germline mutations in CDH1 are infrequent in women with early-onset or familial lobular breast cancers. J Med Genet. 2011;48(1):64-68.

19. Heitzer E, Lax S, Lafer I, et al. Multiplex genetic cancer testing identifies pathogenic mutations in TP53 and CDH1 in a patient with bilateral breast and endometrial adenocarcinoma. BMC Med Genet. 2013;14:129.

20. Corso G, Marrelli D, Pascale V, et al. Frequency of CDH1 germline mutations in gastric carcinoma coming from high- and low-risk areas: metanalysis and systematic review of the literature. BMC Cancer. 2012;12:8. 